\title{
Benzodiazepine-induced superoxide signals B cell apoptosis: mechanistic insight and potential therapeutic utility
}

\author{
Neal B. Blatt, ${ }^{1}$ Jeffrey J. Bednarski, ${ }^{1}$ Roscoe E. Warner, ${ }^{2}$ Francesco Leonetti, ${ }^{3}$ \\ Kathryn M. Johnson, ${ }^{4}$ Anthony Boitano, ${ }^{1}$ Raymond Yung, ${ }^{5}$ Bruce C. Richardson, ${ }^{4,5}$ \\ Kent J. Johnson, ${ }^{2}$ Jonathan A. Ellman, ${ }^{3}$ Anthony W. Opipari, Jr., ${ }^{6}$ and Gary D. Glick ${ }^{1,4}$
}

\author{
${ }^{1}$ Department of Chemistry, and \\ ${ }^{2}$ Department of Pathology, University of Michigan, Ann Arbor, Michigan, USA \\ ${ }^{3}$ Department of Chemistry, University of California, Berkeley, Berkeley, California, USA \\ ${ }^{4}$ Graduate Program in Immunology, \\ ${ }^{5}$ Department of Internal Medicine, and \\ ${ }^{6}$ Department of Obstetrics and Gynecology, University of Michigan, Ann Arbor, Michigan, USA
}

\begin{abstract}
The properties of a proapoptotic 1,4-benzodiazepine, Bz-423, identified through combinatorial chemistry and phenotype screening are described. $\mathrm{Bz}-423$ rapidly generated superoxide $\left(\mathrm{O}_{2}^{-}\right)$in transformed Ramos B cells. This $\mathrm{O}_{2}{ }^{-}$response originated from mitochondria prior to mitochondrial transmembrane gradient collapse and opening of the permeability transition pore. Bz-423-induced $\mathrm{O}_{2}{ }^{-}$functioned as an upstream signal that initiated an apoptotic program characterized by cytochrome $c$ release, mitochondrial depolarization, and caspase activation. Pretreatment of cells with agents that either block the formation of $\mathrm{Bz}-423$-induced $\mathrm{O}_{2}{ }^{-}$or scavenge free radicals attenuated the death cascade, which demonstrated that cell killing by Bz-423 depends on $\mathrm{O}_{2}^{-}$. Parallels between Ramos cells and germinal center $\mathrm{B}$ cells prompted experiments to determine whether $\mathrm{Bz}-423$ had therapeutic activity in vivo. This possibility was tested using the $(\mathrm{NZB} \times \mathrm{NZW}) \mathrm{F}_{1}$ murine model of lupus, in which the pathologically enhanced survival and expansion of germinal center $B$ cells mediate disease. Administration of Bz-423 for 12 weeks specifically controlled germinal center hyperplasia and reduced the histological evidence of glomerulonephritis. Collectively, these studies define a new structure-function relationship for benzodiazepines and point to a new target and mechanism that could be of value for developing improved drugs to manage systemic lupus erythematosus and related disorders.
\end{abstract}

J. Clin. Invest. 110:1123-1132 (2002). doi:10.1172/JCI200216029.

\section{Introduction}

Cytotoxicity is an important mechanism for treating autoimmune diseases like systemic lupus erythematosus

Received for publication May 29, 2002, and accepted in revised form August 13, 2002.

Address correspondence to: Gary D. Glick, Department of Chemistry, University of Michigan, 930 North University Avenue, Ann Arbor, Michigan 48109-1055, USA. Phone: (734) 764-4548; Fax: (734) 615-8902; E-mail: gglick@umich.edu.

Conflict of interest: No conflict of interest has been declared.

Nonstandard abbreviations used: systemic lupus erythematosus (SLE); superoxide $\left(\mathrm{O}_{2}^{-}\right)$; reactive oxygen species (ROS); germinal center $(\mathrm{GC}) ;(\mathrm{NZB} \times \mathrm{NZW}) \mathrm{F}_{1}(\mathrm{NZB} / \mathrm{W})$; tacrolimus (FK506); $\mathrm{N}$-benzoylcarbonyl-Val-Ala-Asp-fluoromethylketone (z-VAD); dihydroethidium (DHE); 3,3'-dihexyloxacarbocyanine iodide $\left(\mathrm{DiOC}_{6}[3]\right.$ ) carboxyfluorescein-Val-Ala-Asp-fluoromethylketone (FAM-VAD-fmk); manganese(III)meso-tetrakis(4-benzoic acid)porphyrin (MnTBAP); propidium iodide (PI); mitochondrial transmembrane potential $\left(\Delta \Psi_{\mathrm{m}}\right)$; carbonyl cyanide $m$ -

chlorophenylhydrazone (CCCP); $2^{\prime}, 7^{\prime}$-dichlorodihydrofluorescin diacetate (DCFH-DA); 2', $7^{\prime}$-dichlorodihydrofluorescin (DCFH); $2^{\prime}, 7^{\prime}$-dichlorofluorescein (DCF); peripheral benzodiazepine receptor (PBR); 1-(2-chlorophenyl)- $N$-methyl- $N$-(1-methylpropyl)3-isoquinolinecarboxamide (PK11195); mitochondrial permeability transition (MPT); mitochondrial respiratory chain (MRC); B cell receptor (BCR).
(SLE) (1) and is the basis for nearly all cancer treatments (2). The selectivity of many cytotoxic agents is limited and generally relies on the differential ability of diseased and healthy cells to tolerate and repair drug-induced cellular damage. Compounds that regulate disease-specific targets clearly have the potential to be better drugs - the development of imatinib mesylate, which inhibits the oncogenic kinase Bcr-Abl, is a recent example of such a molecule (3). However, development of cytotoxic therapies that exploit disease-specific targets remains challenging. For many diseases, suitable targets have not been identified, and in cases where they exist (4), relatively few have been validated to the extent that it is known that blocking function controls disease.

Diversity-oriented synthesis and phenotype screening have been advanced as a robust method for identifying bioactive compounds (5). In this approach, arrays of molecules are synthesized and screened to identify leads based directly on function rather than affinity for a target. As part of efforts to identify new proapoptotic agents, we synthesized a library of 1,4-benzodiazepines and screened it for lymphotoxic members. Benzodiazepines were selected because they are 
amenable to combinatorial synthesis (6), and because under certain conditions, some benzodiazepines influence cell survival (7). Also, because benzodiazepines do not damage DNA or interfere with nucleotide metabolism, cytotoxic benzodiazepines would likely possess unique modes of action.

Our screening experiments identified Bz-423 (Figure 1a). Unlike benzodiazepines with anxiolytic properties, $\mathrm{Bz}-423$ did not bind to the central benzodiazepine receptor. Incubation of transformed Ramos $\mathrm{B}$ cells with $\mathrm{Bz}-423$ rapidly generated superoxide $\left(\mathrm{O}_{2}^{-}\right)$and that this reactive oxygen species (ROS) acted as an upstream signal that commenced an apoptotic process. Cell fractionation experiments revealed that the $\mathrm{O}_{2}^{-}$response resulted from the interaction of $\mathrm{Bz}-423$ and a target within mitochondria. Because Ramos cells model aspects of germinal center (GC) B cell physiology (8), we reasoned that Bz-423 could have activity against GCs in vivo. This hypothesis was tested using the $(\mathrm{NZB} \times \mathrm{NZW}) \mathrm{F}_{1}$ $(\mathrm{NZB} / \mathrm{W})$ model of lupus, in which aberrant survival and expansion of GC B cells drive disease $(9,10)$. Bz-423 controlled GC hyperplasia and the subsequent development of glomerulonephritis in these mice. Collectively, these studies highlight a new role for $\mathrm{O}_{2}^{-}$in $\mathrm{B}$ cell apoptosis and identify Bz-423 as a novel lead for the development of more selective cytotoxic molecules to manage SLE and related disorders.

\section{Methods}

Reagents. Tacrolimus (FK506) was obtained from Fujisawa Pharmaceutical Co. Ltd. (Osaka, Japan). N-benzoylcarbonyl-Val-Ala-Asp-fluoromethylketone (z-VAD) was obtained from Enzyme Systems Products Inc. (Livermore, California, USA). Dihydroethidium (DHE) and 3,3'-dihexyloxacarbocyanine iodide $\left(\operatorname{DiOC}_{6}[3]\right)$ were obtained from Molecular Probes Inc. (Eugene, Oregon, USA). Carboxyfluorescein-Val-Ala-Asp-fluoromethylketone (FAM-VAD-fmk) was purchased from Intergen Co. (Purchase, New Jersey, USA). Manganese(III)mesotetrakis(4-benzoic acid)porphyrin (MnTBAP) was purchased from Alexis Corp. (San Diego, California, USA). Benzodiazepines were synthesized as described (6). Other reagents were obtained from Sigma-Aldrich (St. Louis, Missouri, USA).

Cell lines and culture. Ramos cells were maintained in RPMI supplemented with $10 \%$ heat-inactivated FBS, penicillin $(100 \mathrm{U} / \mathrm{ml})$, streptomycin $(100 \mu \mathrm{g} / \mathrm{ml})$, and L-glutamine $(290 \mu \mathrm{g} / \mathrm{ml})$. In vitro experiments were conducted in media containing $2 \%$ FBS. Organic compounds were dissolved in media containing 0.5\% DMSO.

Detection of cell death and hypodiploid DNA. Cell viability was assessed by staining with propidium iodide (PI; $1 \mu \mathrm{g} / \mathrm{ml}$ ). PI fluorescence was measured using a FACSCalibur flow cytometer (BD Biosciences Inc., San Diego, California, USA). Measurement of hypodiploid DNA was conducted after incubating cells in labeling solution $(50 \mu \mathrm{g} / \mathrm{ml}$ of PI in PBS containing $0.2 \%$ Triton and $10 \mu \mathrm{g} / \mathrm{ml} \mathrm{RNase} \mathrm{A)} \mathrm{at} 4^{\circ} \mathrm{C}$ for 12 hours. Data were analyzed excluding aggregates.
Detection of $\mathrm{O}_{2}^{-}$, mitochondrial transmembrane potential, and caspase activation. To detect $\mathrm{O}_{2}{ }^{-}$, we incubated cells with DHE $(10 \mu \mathrm{M})$ for 30 minutes at $37^{\circ} \mathrm{C}$ and measured ethidium fluorescence by flow cytometry. Flow measurement of the mitochondrial transmembrane potential $\left(\Delta \Psi_{\mathrm{m}}\right)$ was conducted by first labeling cells with $\mathrm{DiOC}_{6}[3](20 \mathrm{nM})$ for 15 minutes at $37^{\circ} \mathrm{C}$. Carbonyl cyanide $m$-chlorophenylhydrazone (CCCP; 50 $\mu \mathrm{M}$ ) was used as a positive control for disruption of $\Delta \Psi_{\mathrm{m}}$. Caspase activation assays were performed with FAM-VAD-fmk. Processing of the substrate was evaluated by flow cytometry.

Subcellular fractionation and cytochrome $c$ detection. Ramos cells $\left(250 \times 10^{6}\right.$ cells per sample) were treated with Bz-423 $(10 \mu \mathrm{M})$ or vehicle for 1-5 hours. Cells were pelleted, resuspended in buffer $(68 \mathrm{mM}$ sucrose, $220 \mathrm{mM}$ mannitol, $10 \mathrm{mM}$ HEPES-NaOH pH 7.4, 10 $\mathrm{mM} \mathrm{KCl}, 1 \mathrm{mM}$ EDTA, $1 \mathrm{mM}$ EGTA, $10 \mu \mathrm{g} / \mathrm{ml}$ leupeptin, $10 \mu \mathrm{g} / \mathrm{ml}$ aprotinin, $1 \mathrm{mM}$ PMSF), incubated on ice for 10 minutes, and homogenized. The homogenate was centrifuged $\left(5\right.$ minutes twice at $4^{\circ} \mathrm{C}$, $800 \mathrm{~g}$ ) to pellet nuclei and debris and again $(15$ minutes at $4^{\circ} \mathrm{C}, 16,000 \mathrm{~g}$ ) to pellet mitochondria. The supernatant was concentrated, electrophoresed on $12 \%$ SDS gels, and transferred to Hybond ECL membranes (Amersham Pharmacia Biotech, Piscataway, New Jersey, USA). After blocking (PBS with 5\% dried milk and $0.1 \%$ Tween), the membranes were probed with an anti-cytochrome $c \mathrm{mAb}$ (Pharmingen, San Diego, California, USA; $2 \mu \mathrm{g} / \mathrm{ml}$ ) followed by an anti-mouse horseradish peroxidase-conjugated secondary antibody with detection by chemiluminescence (Amersham Pharmacia Biotech).

ROS production in isolated mitochondria. Male LongEvans rats were starved overnight and sacrificed by decapitation. Liver samples were homogenized in buffer A $(250 \mathrm{mM}$ sucrose, $10 \mathrm{mM}$ Tris, $0.1 \mathrm{mM}$ EGTA pH 7.4, $\left.4^{\circ} \mathrm{C}\right)$, and nuclei and cellular debris were pelleted (10 minutes, $830 \mathrm{~g}$ ). Mitochondria were collected by centrifugation (10 minutes, $15,000 \mathrm{~g}$ ), and the supernatant was collected as the S15 fraction. The mitochondrial pellet was washed three times with buffer $B(250 \mathrm{mM}$ sucrose, $10 \mathrm{mM}$ Tris $\mathrm{pH}$ 7.4 ), and resuspended in buffer $B$ at $20-30 \mathrm{mg} / \mathrm{ml}$. Mitochondria were diluted $(0.5 \mathrm{mg} / \mathrm{ml})$ in buffer C (200 mM sucrose, $10 \mathrm{mM}$ Tris $\mathrm{pH} 7.4,1 \mathrm{mM} \mathrm{KH}_{2} \mathrm{PO}_{4}$, $10 \mu \mathrm{M}$ EGTA, $2.5 \mu \mathrm{M}$ rotenone, $5 \mathrm{mM}$ succinate as an energy source) containing $2^{\prime}, 7^{\prime}$-dichlorodihydrofluorescin diacetate (DCFH-DA; $1 \mu \mathrm{M})$. For state 3 measurements (maximum mitochondrial respiration rate), ADP ( $2 \mathrm{mM})$ was included in the buffer, and prior to the addition of $\mathrm{Bz}-423$, mitochondria were allowed to charge for 2 minutes. To induce state 4 (minimal respiration rate), oligomycin $(10 \mu \mathrm{M})$ was added to buffer $C$. The oxidation of $2^{\prime}, 7^{\prime}$-dichlorodihydrofluorescin (DCFH) to $2^{\prime}, 7^{\prime}$-dichlorofluorescein (DCF) was monitored at $37^{\circ} \mathrm{C}$ with a spectrofluorometer $\left(\lambda_{\mathrm{ex}}\right.$ [emission wavelength]: $503 \mathrm{~nm} ; \lambda_{\mathrm{em}}$ [excitation wavelength]: $522 \mathrm{~nm}$ ). ROS production after 
the induction phase was evaluated by measurement of the slope of each curve by linear regression. To detect effects on $\mathrm{O}_{2}{ }^{-}$and $\Delta \Psi_{\mathrm{m}}$, mitochondria were incubated for 15 minutes at $37^{\circ} \mathrm{C}$ in buffer $\mathrm{C}$ with vehicle, Bz-423, or CCCP containing DHE $(5 \mu \mathrm{M})$ or DiOC $_{6}[3](20 \mathrm{nM})$, and aliquots were analyzed by fluorescence microscopy.

Animals and drug delivery. Female NZB/W mice (The Jackson Laboratory, Bar Harbor, Maine, USA) were randomly distributed into treatment and control groups. Through intraperitoneal injections, control mice received vehicle ( $50 \mu \mathrm{l}$ aqueous DMSO) and treatment mice received $\mathrm{Bz}-423$ dissolved in vehicle $(60 \mathrm{mg} / \mathrm{kg})$. Peripheral blood was obtained from the tail veins for the preparation of serum. Samples of the spleen and kidney were preserved in $10 \%$ formalin or by snap-freezing. Additional spleen sections from each animal were used to prepare single-cell suspensions.

Flow cytometric analysis of splenocytes. Splenocytes were prepared by mechanical disruption and red blood cells removed by isotonic lysis. Cells were stained at $4^{\circ} \mathrm{C}$ with fluorescent-conjugated anti-Thy1.2 (Pharmingen; $1 \mu \mathrm{g} / \mathrm{ml}$ ) and/or anti-B220 (Pharmingen; $1 \mu \mathrm{g} / \mathrm{ml}$ ) for 15 minutes. To detect outer-membrane phosphatidyl serine, cells were incubated with FITC-conjugated annexin V and PI (Roche Molecular Biochemicals, Indianapolis, Indiana, USA; $1 \mu \mathrm{g} / \mathrm{ml}$ ).

In vivo determination of ROS. Spleens were removed from 4-month-old NZB/W mice treated with Bz-423 or vehicle, and frozen. ROS production was measured using manganese(II)3,3,9-diaminobenzidine as described (11).

Histology. Formalin-fixed kidney sections were stained with hematoxylin and eosin (H\&E), periodic acid-Schiff, and Masson's trichrome. Glomerular immune complex deposition was detected by immunofluorescence using frozen tissue stained with FITC-conjugated goat anti-mouse IgG (Southern Biotechnology Associates, Birmingham, Alabama, USA). Sections were analyzed blinded for nephritis and IgG deposition using a 0 -to-4+ scale. The degree of lymphoid hyperplasia was scored on a 0-to-4+ scale using spleen sections stained with H\&E. To identify B cells, sections were stained with biotinylated antiB220 (Pharmingen; $1 \mu \mathrm{g} / \mathrm{ml}$ ) followed by streptavidin-Alexa 594 (Molecular Probes Inc.; $5 \mu \mathrm{g} / \mathrm{ml}$ ). Frozen spleen sections were analyzed for TUNELpositive cells using an In Situ Cell Death Detection kit (Roche Molecular Biochemicals) and were scored on a 0 -to- $4+$ scale.

IgG titers, blood urea nitrogen, and proteinuria. Anti-DNA and IgG titers were determined by ELISA (12). Serum blood urea nitrogen was measured by the University of Michigan Hospital. Proteinuria was measured using ChemStrip 6 (Roche Molecular Biochemicals).

Statistical analysis. Statistical significance was assessed using the Mann-Whitney $U$ test unless otherwise noted. $P$ values are one-tailed, and data are given as mean \pm SEM.

\section{Results}

$B z-423$ induces cell death in vitro. The activity of $\mathrm{Bz}-423$ was first compared with that of ligands of the peripheral benzodiazepine receptor (PBR), an $18-\mathrm{kDa}$ transmembrane protein located in the mitochondrial membrane, because some ligands of the PBR are thought to modulate death signals from mitochondria (7). Both 1-(2-chlorophenyl)- $N$-methyl- $N$ (1-methylpropyl)-3-isoquinolinecarboxamide (PK11195) and 4'-chlorodiazepam bind tightly to the $\operatorname{PBR}\left(K_{\mathrm{d}}=0.3\right.$ and $30 \mathrm{nM}$, respectively; ref. 7$)$ but are only cytotoxic at more than ten times the $\mathrm{ED}_{50}$ of Bz-423 (Figure 1b). Competition assays for the PBR demonstrated that a 1,000 -fold excess of $\mathrm{Bz}-423$ was necessary to reduce $\left[{ }^{3} \mathrm{H}\right]$-PK11195 binding by $50 \%$, and preincubation of cells with excess PK11195 (>20 $\mu \mathrm{M}$ ) did not block the activity of Bz-423 (our unpublished data). These data indicate that cell killing by $\mathrm{Bz}-423$ does not result from binding to the PBR. The activity of $\mathrm{Bz}-423$ depends on its structure, since deleting either the naphthyl $(\triangle \mathrm{NAP})$ or the phenolic hydroxyl $(\Delta \mathrm{OH})$ group, elements that distinguish Bz-423 from diazepam, dramatically reduced cytotoxic activity (Figure 1b).

Bz-423-induced cytotoxicity was characterized by cell shrinkage, nuclear condensation, cytoplasmic vacuolization, membrane blebbing, and DNA fragmentation (hypodiploid DNA; Figure 1, c and d), consistent with apoptosis. These data also show that $\mathrm{Bz}-423$ affects cell cycle progression, since there were fewer cells in the $\mathrm{G}_{2} / \mathrm{M}$ phase (Figure 1d). To probe the role of caspases in Bz-423 killing, cells were preincubated with $z-V A D$, an irreversible caspase inhibitor. $z-V A D$ completely prevented $\mathrm{Bz}-423$-mediated apoptosis as measured by DNA fragmentation. Less than $5 \%$ of cells treated with z-VAD and $\mathrm{Bz}-423$ had hypodiploid DNA, compared with $69 \%$ of cells treated with Bz- 423 alone (Figure 1d). Inhibiting caspase activity did not protect against cell death; cellular morphology demonstrated that Bz-423 still killed more than $80 \%$ of cells in cultures with z-VAD (Figure 1c). Similar results have been reported for other apoptotic stimuli, where blocking caspase activity prevents DNA fragmentation but not cell death (13).

Mediators of Bz-423-induced apoptosis. To characterize the death mechanism engaged by $\mathrm{Bz}-423$, intracellular ROS, $\Delta \Psi_{\mathrm{m}}$, cytochrome $c$ release, caspase activation, and DNA fragmentation were measured over time. We chose these endpoints as they have been previously implicated in B cell apoptosis (14). The first event detected after exposure to Bz-423 was an increase in the fraction of cells that stained with DHE, a redox-sensitive agent that reacts specifically with $\mathrm{O}_{2}^{-}$(15) (Figure 2a). Levels of $\mathrm{O}_{2}^{-}$diminished after an early maximum at 1 hour and then increased again after 4 hours of continued treatment (Figure 2b). This bimodal pattern points to a cellular mechanism limiting $\mathrm{O}_{2}{ }^{-}$and suggests that the "early" and "late" $\mathrm{O}_{2}^{-}$maxima result from different processes. 


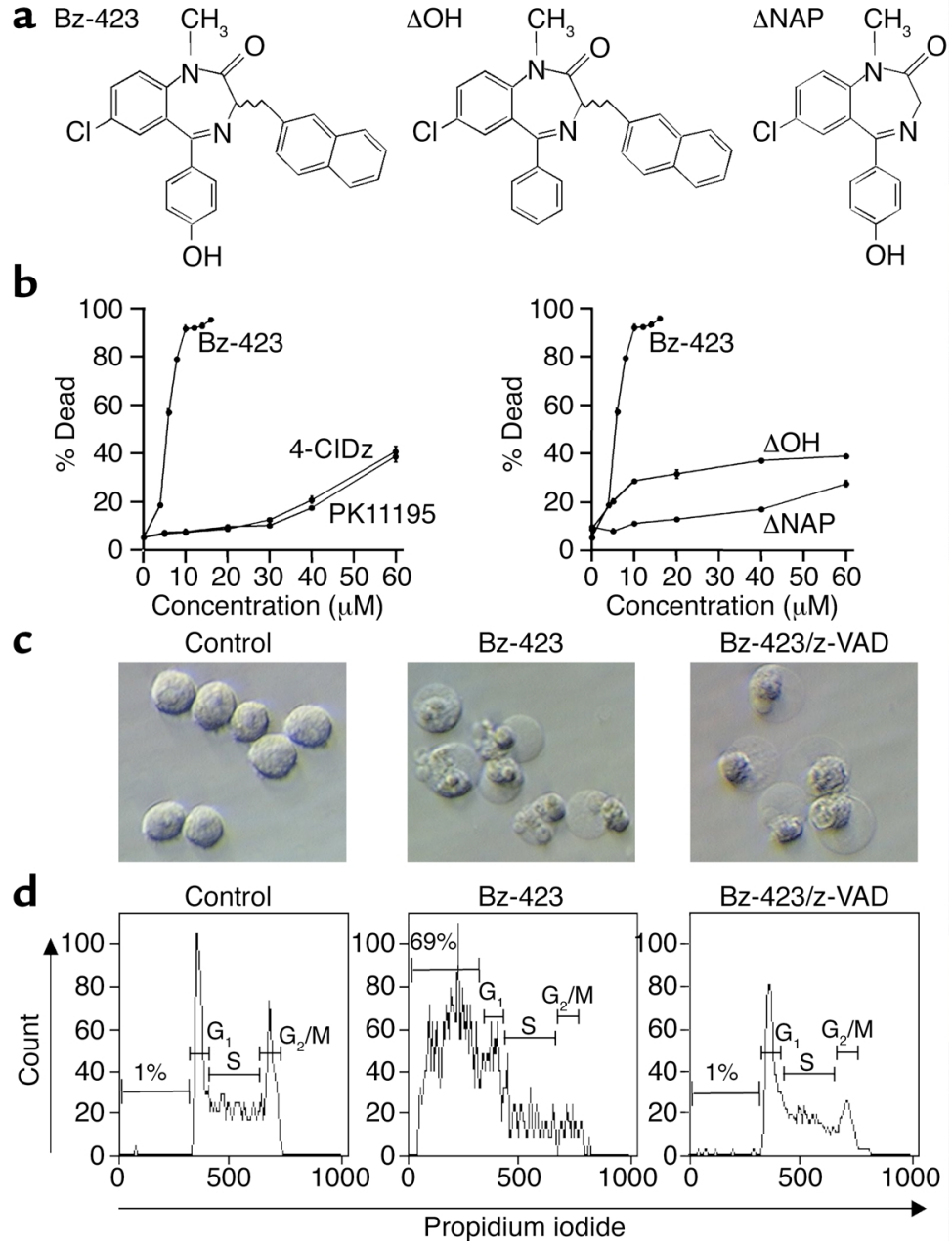

Figure 1

(a) Structure of Bz-423 and inactive congeners. (b) Effect of Bz-423, 4'-chlorodiazepam (4-CIDz), PK11195, $\triangle \mathrm{NAP}$, and $\Delta \mathrm{OH}$ on Ramos cell viability at 24 hours, determined by PI permeability. The $\mathrm{ED}_{50}$ of $\mathrm{Bz}-423$ is $4 \mu \mathrm{M}$. (c) Interference contrast micrographs $(\times 400)$ of cells treated for 24 hours with vehicle, Bz-423 (10 $\mu \mathrm{M})$, or Bz-423 (10 $\mu \mathrm{M})$ plus z-VAD $(100 \mu \mathrm{M})$. (d) After treatment as in c, cells were analyzed by flow cytometry to detect DNA content; percentages indicate fraction of cells with hypodiploid (apoptotic) DNA. Distribution of nonapoptotic cells in $\mathrm{G}_{1}, \mathrm{~S}$, and $\mathrm{G}_{2} / \mathrm{M}$ phases was altered by $\mathrm{Bz}-423$ (33\%, 42\%, and $24 \%$ for control vs. $53 \%, 38 \%$, and $8 \%$ for Bz-423). Pretreatment with z-VAD blocked formation of hypodiploid DNA and partially restored cell cycle distribution. All panels are representative of more than five separate determinations. release of cytochrome $c$, suggesting that the secondary rise in $\mathrm{O}_{2}^{-}$results from these processes (18). Caspase activation, measured by processing of the pan-caspase-sensitive fluorescent substrate FAM-VADfmk, tracked $\Delta \Psi_{\mathrm{m}}$, whereas the appearance of hypodiploid DNA was slightly delayed with respect to caspase activation (Figure $2 \mathrm{~b})$. Collectively, these results indicate that $\mathrm{Bz}-423$ induces a mitochondria-dependent apoptotic pathway.

$B z-423$ directly targets mitochondria. Since the early $\mathrm{O}_{2}-$ precedes other cellular events, it is possible that this ROS has a regulatory role. In nonphagocytic cells, redox enzymes, along with the mitochondrial respiratory chain (MRC), are the primary sources of ROS (19). The MRC, which is a group of multiprotein electron transport assemblies (complexes I-IV), reduces molecular oxygen to water. This process establishes a proton gradient across the inner mitochondrial membrane, providing energy for ATP synthesis by the mitochondrial $\mathrm{F}_{0} \mathrm{~F}_{1}$-ATPase (complex V). To determine the basis for this response, we assayed inhibitors of these systems for their ability to regulate $\mathrm{Bz}-423$-induced $\mathrm{O}_{2}^{-}$(at 1 hour) (Table 1). Of these reagents, only sodium azide, which acts primarily on cytochrome $c$ oxidase (complex IV) (20), and micromolar amounts of FK506, which block the formation of $\mathrm{O}_{2}^{-}$by the ubiquinolcytochrome $c$ reductase component of MRC complex III (21), modulated Bz-423. These findings suggest that mitochondria are the source of $\mathrm{Bz}-423$-induced $\mathrm{O}_{2}{ }^{-}$and that a component of the MRC is involved in the response. Although the inhibition by FK506 could result from binding to either calcineurin or FK506-binding proteins, natural products that bind tightly to these proteins (rapamycin and cyclosporin $\mathrm{A}$, respectively) did not diminish the $\mathrm{Bz}-423$ $\mathrm{O}_{2}{ }^{-}$response (Table 1).

Collapse of $\Delta \Psi_{\mathrm{m}}$ was detected using $\operatorname{DiOC}_{6}[3]$, a mitochondria-selective potentiometric probe (16). The gradient change began after the early $\mathrm{O}_{2}{ }^{-}$ response and was observed in more than $90 \%$ of cells by 5 hours. Cytochrome $c$ release from mitochondria, a key step enabling caspase activation (17), was studied by immunoblotting cytosolic fractions. Levels of cytosolic cytochrome $c$ above amounts in cells treated with vehicle were detected by 5 hours (inset, Figure $2 \mathrm{~b}$ ). This release was coincident with the disruption of $\Delta \Psi_{\mathrm{m}}$, and, together, these results are consistent with opening of the mitochondrial permeability transition (MPT) pore. Indeed, the late increase in $\mathrm{O}_{2}{ }^{-}$tracked with the $\Delta \Psi_{\mathrm{m}}$ collapse and the
$\mathrm{O}_{2}^{-}$production by $\mathrm{Bz}-423$ could result from binding to a protein within mitochondria or a target in another compartment that signals mitochondria to generate ROS. To distinguish between these alternatives, isolated rat liver mitochondria were assayed for ROS production by monitoring of the oxidation of DCFH-DA to DCF in the presence and absence of $\mathrm{Bz}-423$ as previously described (22). In this assay, the rate of DCF production increased after a lag period of about $15 \mathrm{~min}$ utes, during which endogenous reducing equivalents were consumed and the acetate moieties on the probe were hydrolyzed to yield DCFH, the redox-active species. Under aerobic conditions supporting state 3 respiration (succinate as energy plus ADP), both 

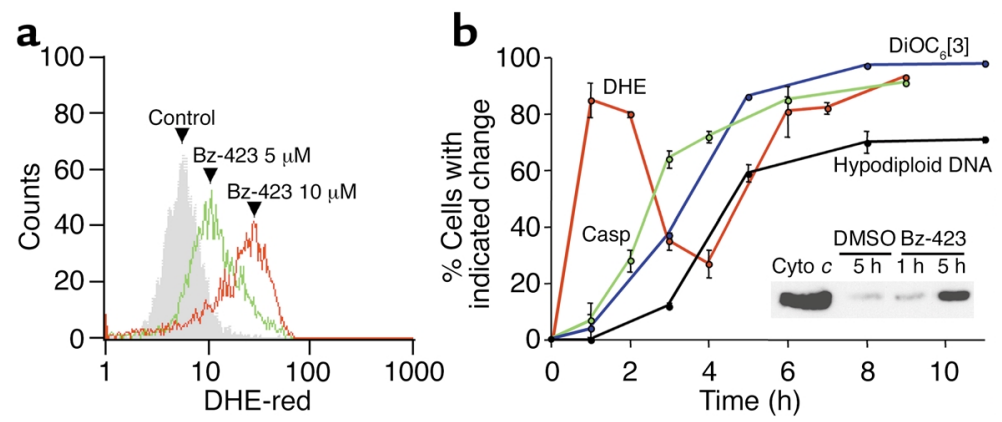

Figure 2

Mediators of Bz-423-induced apoptosis. (a) Ramos cells were incubated with vehicle or Bz-423 for 1 hour. Fluorescence intensity increased above that of control (shaded histogram) with 5 and $10 \mu \mathrm{M} \mathrm{Bz}-423$ (green and red histograms, respectively). (b) Time course of changes detected in Ramos cells upon treatment with Bz-423 (10 $\mu \mathrm{M})$. Data are presented as percent cells with indicated response relative to time-matched vehicle controls. No changes in cells treated with vehicle during this time frame were observed. The error on the $\mathrm{DiOC}_{6}[3]$ curve is sufficiently low that the error bars are not visible. Inset: Cytochrome $c$ release. First lane: purified cytochrome $c(20 \mathrm{ng})$; second lane: cytosolic fraction isolated 5 hours after incubation with vehicle; third and fourth lanes: cytosolic fractions isolated 1 and 5 hours after incubation with Bz-423, respectively. $\mathbf{a}$ and $\mathbf{b}$ represent data from more than five separate determinations.

antimycin $\mathrm{A}$, which generates $\mathrm{O}_{2}^{-}$by inhibiting ubiquinol-cytochrome $c$ reductase, and $\mathrm{Bz}-423$ increased the rate of ROS production nearly twofold after the induction phase, based on comparison of the slopes of each curve to control (22) (Figure 3a). Swelling was not observed, demonstrating that $\mathrm{Bz}-423$ does not directly target the MPT pore (13) (Figure 3b). Neither Bz-423 nor antimycin A generated substantial ROS in the subcellular S15 fraction (cytosol and microsomes; Figure 3c), and Bz-423 did not stimulate ROS if mitochondria were in state 4 (succinate plus oligomycin), even though antimycin $\mathrm{A}$ was active under these conditions (Figure 3d). Together, these experiments demonstrate that mitochondria contain a molecular target for $\mathrm{Bz}-423$, and that state 3 respiration is required for the $\mathrm{O}_{2}{ }^{-}$response.
To relate data obtained with mitochondria to observations in whole cells, we probed isolated mitochondria with DHE and $\mathrm{DiOC}_{6}[3]$. In both state 3 and state 4, control mitochondria fluoresced brightly with $\mathrm{DiOC}_{6}[3]$, indicating that $\Delta \Psi_{\mathrm{m}}$ was intact (Figure $3 \mathrm{e}$ ). Addition of $\mathrm{Bz}-423$ did not alter the $\mathrm{DiOC}_{6}[3]$ signal over the course of measurement, indicating that $\Delta \Psi_{\mathrm{m}}$ had not collapsed. In contrast, the protonophore CCCP disrupted $\Delta \Psi_{\mathrm{m}}$, and this abolished the green fluorescence. Oxidation of DHE by endogenous $\mathrm{O}_{2}{ }^{-}$produces red fluorescence within mitochondria. Bz-423 did not induce $\mathrm{O}_{2}{ }^{-}$over control levels in state 4. However, DHE fluorescence was markedly increased after incubation of mitochondria with $\mathrm{Bz}-423$ under state 3 conditions, reflecting elevated levels of $\mathrm{O}_{2}{ }^{-}$. Collectively, these experiments confirmed the results of the DCF assay, demonstrated an ROS response in mitochondria consistent with that seen in whole cells, and provided evidence to support the hypothesis that the early $\mathrm{O}_{2}{ }^{-}$does not result from collapse of $\Delta \Psi_{\mathrm{m}}$ (see Figure $2 \mathrm{~b}$ ).

Bz-423-induced apoptosis depends on the early $\mathrm{O}_{2}$ response. To determine whether $\mathrm{O}_{2}^{-}$is needed for $\mathrm{Bz}-423$-induced apoptosis, we explored the dependency of the apoptotic process on ROS. Pretreatment of cells with FK506, which prevents formation of Bz-423-induced $\mathrm{O}_{2}^{-}$, significantly inhibited caspase activation, mitochondrial depolarization, DNA fragmentation, and cell death (Figure 4a). Moreover, after cells were incubated with Bz-423 for approximately 1 hour, which is the point at which the early $\mathrm{O}_{2}$ response is maximal (see Figure $2 \mathrm{~b}$ ), addition of FK506 was much less effective at blocking cell death (Figure 4b). Preincubating cells with vitamin E, an

Table 1

Effect of ROS inhibitors on the activity of Bz-423

\begin{tabular}{|c|c|c|c|c|}
\hline Target & Inhibitor ${ }^{A}$ & $\begin{array}{l}\text { \% DHE-positive: } \\
\text { inhibitor alone }\end{array}$ & $\begin{array}{l}\text { \% DHE-positive: } \\
\text { Bz-423 plus inhibitor }\end{array}$ & Relative response ${ }^{B}$ \\
\hline & DMSO control & 7 & 72 & $100 \pm 13$ \\
\hline MRC & Sodium azide (1 mM) & 0 & 0 & $1 \pm 1$ \\
\hline MRC/Calcineurin & FK506 $(1 \mu \mathrm{M})$ & 1 & 10 & $14 \pm 10$ \\
\hline FK506-binding proteins & Rapamycin $(1 \mu \mathrm{M})$ & 1 & 60 & $83 \pm 2$ \\
\hline Calcineurin & Cyclosporin A $(0.5 \mu \mathrm{M})$ & 2 & 60 & $83 \pm 2$ \\
\hline Cytochrome P450s & Benzylimidazole $(100 \mu \mathrm{M})$ & 14 & 90 & $126 \pm 7$ \\
\hline Cytochrome P450s & SKF525A $(5 \mu \mathrm{M})$ & 12 & 92 & $128 \pm 6$ \\
\hline Cytochrome P450 (CYP231) & Cimetidine $(100 \mu \mathrm{M})$ & 6 & 83 & $116 \pm 7$ \\
\hline Cyclooxygenase & Indomethacin $(100 \mu \mathrm{M})$ & 4 & 69 & $97 \pm 4$ \\
\hline Monoaminoxidase & Phenelzine $(10 \mu \mathrm{M})$ & 5 & 78 & $109 \pm 4$ \\
\hline Xanthine oxidase & Allopurinol $(100 \mu \mathrm{M})$ & 6 & 79 & $111 \pm 7$ \\
\hline
\end{tabular}

${ }^{A}$ Data are normalized to the response of $\mathrm{Bz}-423$ alone. ${ }^{B}$ Rotenone, myxothiazol, thenoyltrifluoroacetone, antimycin $\mathrm{A}$, stigmatellin, and oligomycin, which inhibit other components of the MRC, along with the flavoenzyme inhibitor diphenyleneiodonium, are less informative, as they independently generate significant ROS in Ramos cells. 

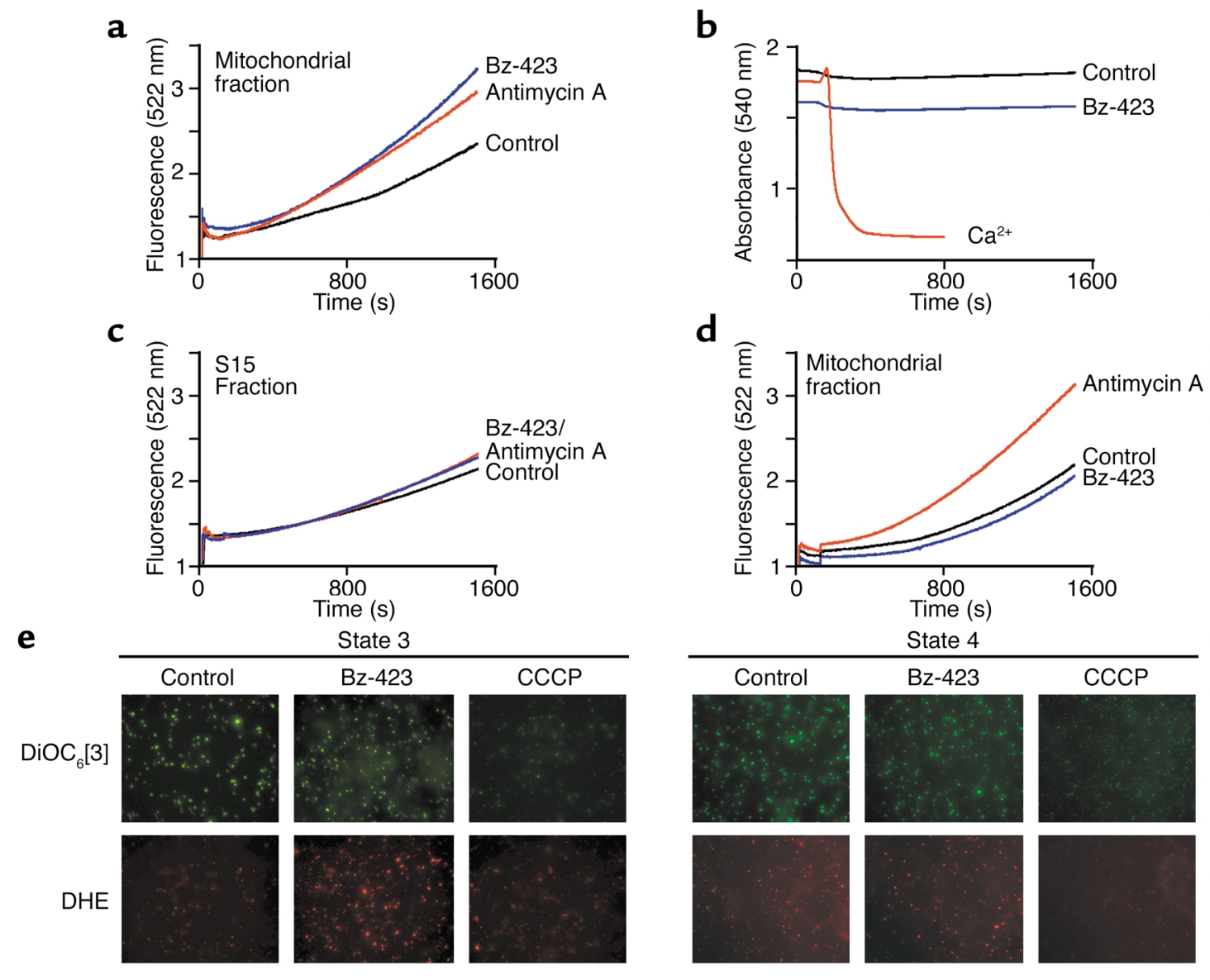

\section{Figure 3}

Bz-423 generates ROS but not swelling in isolated mitochondria. Measurements were conducted in duplicate as described in Methods and represent data obtained with mitochondria from four different rats. Within a given run, a larger slope corresponds to greater ROS production. For these experiments, antimycin A was added at $0.5 \mu \mathrm{M}$ and Bz-423 at $10 \mu \mathrm{M}$. The $r^{2}$ values for the linear regression analysis of the data in a, $\mathbf{c}$, and $\mathbf{d}$ are greater than 0.995. (a) ROS measured in state 3 respiration buffer. The slopes of each curve after the induction phase (1,200-1,500 seconds) are $1.5 \times 10^{-3} \pm \leq 15 \%, 2.1 \times 10^{-3} \pm \leq 15 \%$, and $1.1 \times 10^{-3} \pm \leq 15 \%$ for antimycin A, Bz-423, and vehicle, respectively. $P<0.05$ for vehicle vs. Bz-423 or antimycin A (Student's $t$ test). (b) Swelling of mitochondria in state 3 buffer. Only Ca ${ }^{2+}(400 \mu M)$ triggered the MPT pore. (c) ROS measured using the S15 fraction in state 3 buffer. Slopes are $0.9,0.8$, and $1.0\left(\times 10^{-3} \pm \leq 15 \%\right)$ for antimycin A, $\mathrm{Bz}-423$, and vehicle, respectively. (d) ROS measured in state 4 respiration buffer. Slopes are $2.1,1.3$, and $1.3\left(\times 10^{-3} \pm \leq 15 \%\right)$ for antimycin $\mathrm{A}, \mathrm{Bz}-423$, and vehicle, respectively. $P=0.25$ for vehicle vs. Bz-423; $P=0.035$ for vehicle vs. antimycin A (Student's $t$ test). (e) Micrographs $(\times 630)$ of mitochondria stained with $\mathrm{DHE}$ (red) to detect $\mathrm{O}_{2}{ }^{-}$or with $\mathrm{DiOC}_{6}[3]$ (green) to determine $\Delta \Psi_{\mathrm{m}}$. Each spot represents an individual mitochondrion staining with the indicated dye.

antioxidant that scavenges ROS, or MnTBAP, an $\mathrm{O}_{2}^{-}$ dismutase mimetic, also attenuated each of these endpoints (Figure 4a). MnTBAP was somewhat less effective at influencing the downstream effectors engaged by Bz-423, because dismutation of $\mathrm{O}_{2}{ }^{-}$yields $\mathrm{H}_{2} \mathrm{O}_{2}$, which also triggers the same effectors (23) (Figure $4 a)$. In sum, these data demonstrate that cell killing by Bz-423 depends on $\mathrm{O}_{2}^{-}$.

$B z-423$ induces ROS and kills primary $B$ lymphocytes in vivo. In the NZB/W model of SLE, splenic GC B cells are hyperactivated and pathologically expanded. Somatic hypermutation within GCs produces autoantibodies, a subset of which contribute to the development of glomerulonephritis $(9,24,25)$. Because Ramos cell behavior parallels many of the responses of GC B cells (8), we reasoned that $\mathrm{Bz}-423$ might have activity in NZB/W mice that could be therapeutically useful. To characterize the effect of Bz-423 on NZB/W lymphocytes, we gave mice a single dose of either Bz-423 $(60 \mathrm{mg} / \mathrm{kg} ; n=4)$ or vehicle $(n=4)$ and sacrificed them after $2 \mathrm{~h}$. This dose provides peak serum levels of approximately $5 \mu \mathrm{M} 1$ hour after injection; this is near the $\mathrm{ED}_{50}$ in vitro (see Figure 1b). As shown in Figure 5, a and b, Bz-423 induced robust ROS production in the spleen compared with control. Based on these findings, we administered Bz-423 $(60 \mathrm{mg} / \mathrm{kg} / \mathrm{d} ; n=7)$ or vehicle $(n=7)$ for 7 days to determine whether it was lymphotoxic. A short time frame was chosen so that cellular recruitment or proliferation would not significantly alter splenocyte populations. Total lymphocyte viability in the Bz-423-treated animals after sacrifice was decreased $(81 \% \pm 1 \%$ vs. $85 \% \pm 1 \%$; $P<0.05)$ and $B$ cell apoptosis was increased versus 
controls ( $35 \pm 2$ vs. $27 \pm 2 ; P<0.05)$. These changes were relatively small because early apoptotic cells are rapidly cleared by the reticuloendothelial system in vivo (16). An increase in $\mathrm{T}$ cell apoptosis was not observed $(15 \pm 2$ for both control and treatment; $P=0.23$ ). However, since the kinetics of death and clearance may differ between $\mathrm{B}$ and $\mathrm{T}$ lymphocytes, we cannot exclude the possibility that $\mathrm{Bz}-423$ also kills $\mathrm{T}$ cells in vivo.

$B z-423$ reduces lymphoproliferative and autoimmune disease in $N Z B / W$ mice. We next designed a study to determine whether $\mathrm{Bz}-423$ alters the progression of disease in $\mathrm{NZB} / \mathrm{W}$ mice. Our endpoints were measures of GC B cell hyperplasia, glomerulonephritis, and autoantibody titers, which are established markers of disease (25). Since $\mathrm{Bz}-423$ is a first-generation agent with an unknown toxicological profile, we did not employ mortality as an endpoint. In the protocol, female $\mathrm{NZB} / \mathrm{W}$ mice were given $\mathrm{Bz}-423(60 \mathrm{mg} / \mathrm{kg}$ every other day; $n=25)$ or vehicle (aqueous DMSO; $n=20$ ) beginning at 6.5 months of age, and all were sacrificed 12 weeks latter. This time frame begins after the histological onset of disease and continues to when severe nephritis is usually observed (25).

Disease-related GC hyperplasia in NZB/W mice expanded the white pulp and distorted normal splenic architecture (25). Analysis of spleen sections after termination of the study (12 weeks of dosing) revealed a reduction in the white pulp in mice receiving $\mathrm{Bz}-423$ ( 1 to $2+$ vs. 3 to $4+; P=0.02$ ). Mice given Bz-423 had $40 \%$ fewer GCs relative to controls $\left(10 \pm 2\right.$ vs. $17 \pm 1$ per $\left.10 \mathrm{~mm}^{2} ; P=0.009\right)$, and the GCs in the treated mice were $40 \%$ smaller than those in controls $\left(20 \times 10^{3} \pm 2 \times 10^{3}\right.$ vs. $35 \times 10^{3} \pm 5 \times 10^{3}$ $\mu^{2} ; P=0.013$; Figure 5, c and d). Spleens from $\mathrm{Bz}-423$-treated animals also had more TUNEL-positive cells than did spleens from controls (about $3+$ vs. $1+; P=0.038$ ), and these cells were located within GCs (Figure 5, e and f), suggesting that the decrease in GCs may have resulted from increased apoptosis within this compartment. In addition to affecting GCs, treatment afforded a small decrease in follicular zone B cells $(12 \% ; P=0.046)$ with no clear changes to the marginal zone (data not shown).

To further investigate the changes observed histochemically, splenocytes obtained after termination of the study were analyzed by flow cytometry. As expected, Bz-423 mice had fewer B cells relative to controls (percent B220 splenocytes, $59 \% \pm 3 \%$ vs. $67 \% \pm 2 \%$; $P=0.03$ ) with no apparent effect on $\mathrm{T}$ cells (percent Thy1.2+ splenocytes, Bz- $423,16 \% \pm 2 \%$; control, $17 \% \pm 2 \%$; $P=0.5)$. As discussed above, differences in the rate of apoptosis or clearance between lymphocytes could mask an effect on $T$ cells. Dual staining with combinations of antiB220, - IgM, and -CD5 revealed that no one B cell population (defined by these markers) was altered to a greater extent than any other. Similarly, staining to detect CD4 or CD8, alone or in combination with CD69, CD45RB, CD44, or CD5, showed no specific differences in splenic $\mathrm{T}$ cell populations (data not shown).

During the study, two control mice died with 4+ nephritis, whereas none did in the $\mathrm{Bz}-423$ mice. Based on histology, $60 \%$ of controls (12 of 20) had nephritis of $2+$ or greater, while only $16 \%$ of Bz-423treated mice ( 4 of 25$)$ had disease $\left(\chi^{2}: P=0.003\right)$. At the end of the study, disease in the controls was characterized by diffuse, proliferative glomerulonephritis with expansion of all cellular elements and occasional wire-loop formation, consistent with an average score of $3+$ (Figure 5, g and h). In contrast, Bz-423 mice had milder changes $(1+; P=0.002)$ and less glomerular IgG deposition (1+ in Bz-423 vs. $3+$ in controls; $P=0.002$; Figure 5 , $i$ and $j$ ). At the end of the study, $83 \%$ of controls had abnormally high blood urea nitrogen $(\geq 30 \mathrm{mg} / \mathrm{dl})$, compared with $27 \%$ in the treatment group $\left(\chi^{2}: P=0.001\right)$. A similar trend was observed with proteinuria: $39 \%$ of controls had significant proteinuria $(>100 \mathrm{mg} / \mathrm{dl})$ compared with $18 \%$ of Bz-423-treated mice $\left(\chi^{2}: P=0.1\right)$. Collectively, these data suggest that mice administered $\mathrm{Bz}-423$ had less disease than controls.

To determine the effect of Bz-423 on antibody levels, serum was drawn after $3,6,9$, and 12 weeks of dosing and anti-DNA and IgG titers were measured. After 3 weeks, Bz-423 mice had modestly lower anti-ssDNA $(B z-423,546 \pm 91 \mathrm{U} / \mathrm{ml}$; control, $804 \pm 95 \mathrm{U} / \mathrm{ml}$; $P=0.014)$ and $\mathrm{IgG}(\mathrm{Bz}-423,4.2 \pm 0.3 \mathrm{mg} / \mathrm{ml}$; control,

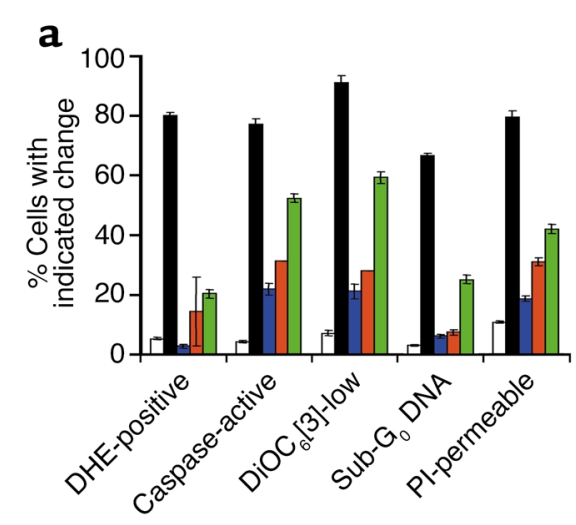

b

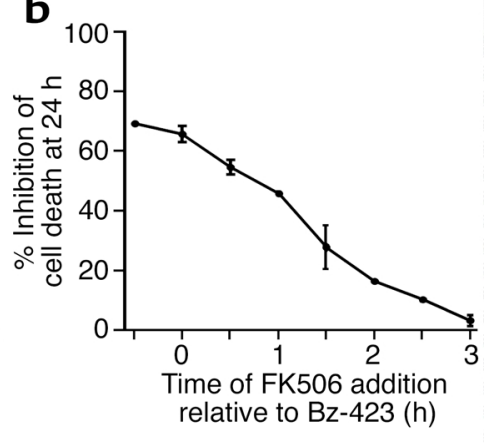

\section{Figure 4}

Regulation of ROS blocks Bz-423-induced killing. (a) After preincubation of Ramos cells for 30 minutes with FK506 (1 $\mu \mathrm{M}$, blue), vitamin E (100 $\mu \mathrm{M}$, red), MnTBAP (100 $\mu \mathrm{M}$, green), or no inhibitor (black), Bz-423 (10 $\mu \mathrm{M})$ was added to the cultures. White bars indicate control samples treated with vehicle alone. Using flow cytometry, DHE fluorescence $\left(\mathrm{O}_{2}^{-}\right)$was measured at 1 hour, caspase activation at 5 hours, $\Delta \Psi_{\mathrm{m}}\left(\mathrm{DiOC}_{6}[3]\right)$ at 5 hours, and PI permeability and hypodiploid DNA at 24 hours. (b) Effect on cell viability of addition of FK506 at times relative to the addition of Bz-423. The results in a and $\mathbf{b}$ represent more than five separate determinations. $P<0.05$ for the differences between Bz-423 and control for each inhibitor at each endpoint (Student's $t$ test). 

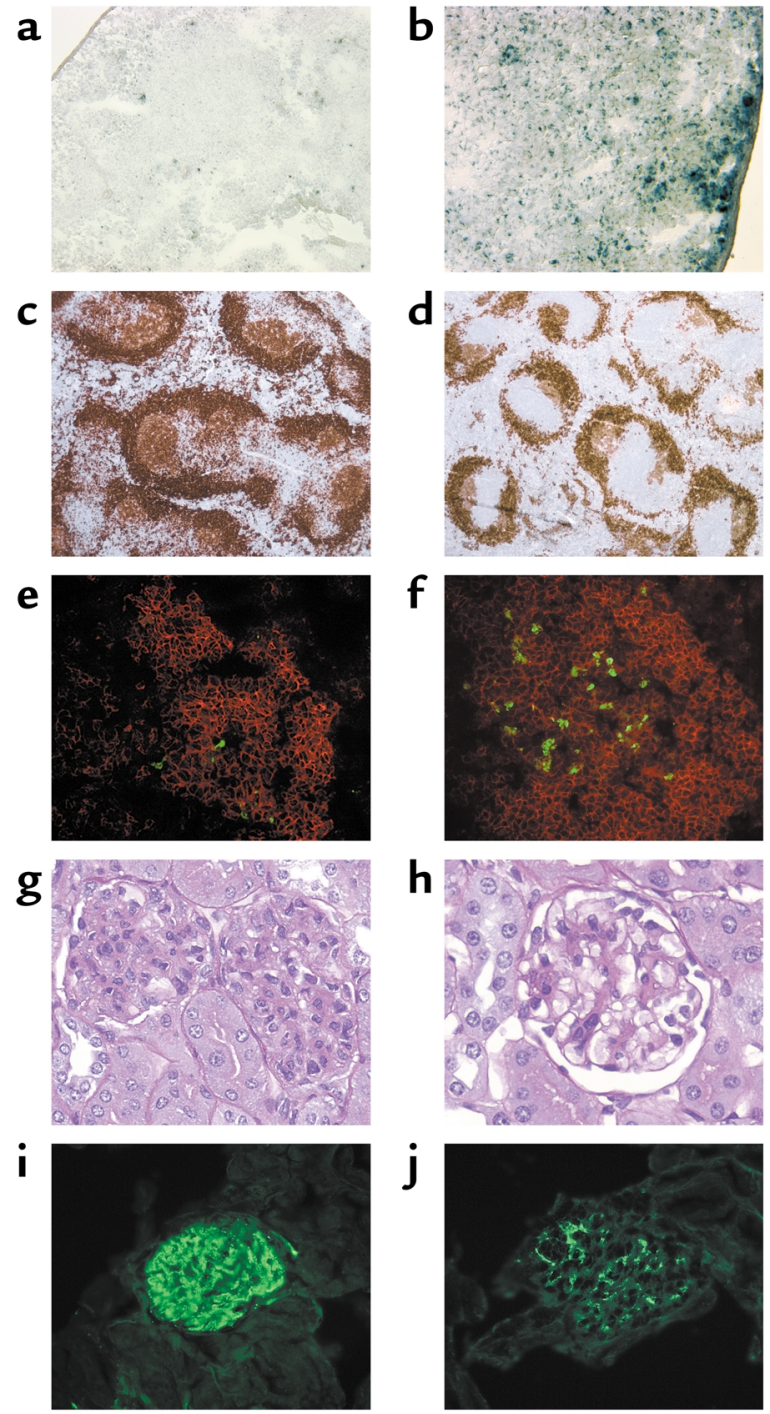

\section{Figure 5}

Splenic and renal histology. Sections showing ROS in NZB/W spleen tissue 2 hours after administration of (a) vehicle or (b) Bz-423 $(\times 100)$. Increased staining was observed in $\mathbf{b}$ compared with $\mathbf{a}$, demonstrating the ROS response. (c and d) Representative histology from 9.5-month-old mice, obtained after terminating longitudinal study. Spleen sections $(\times 50)$ are shown from control (c) and Bz-423 (d) mice stained for B cells with anti-B220 (brown). (e and f) GCs $(\times 400)$ from control (e) and Bz-423 (f) mice were identified by staining with Alexa 594-conjugated anti-B220 (red), and fragmented DNA (TUNEL, green) was detected by indirect immunofluorescence. GC $B$ cells were distinguished by the intensity and location of the B220 staining relative to the splenic follicle architecture and confirmed by staining of serial sections with peanut agglutinin. (g and h) Kidney sections $(\times 400)$ from control (g) and Bz-423 (h) mice stained with periodic acid-Schiff, showing representative glomeruli. (i and $\mathbf{j}$ ) Kidney sections $(\times 400)$ from control $(\mathbf{i})$ and Bz-423 (j) mice, visualized with FITC-conjugated anti-lg.

$6.4 \pm 0.6 \mathrm{mg} / \mathrm{ml} ; P=0.05)$, whereas anti-dsDNA levels also trended lower $(\mathrm{Bz}-423,463 \pm 94 \mathrm{U} / \mathrm{ml}$; control, $640 \pm 117 \mathrm{U} / \mathrm{ml} ; P=0.083)$. At later times, differences between the control and treatment groups were not observed (after 12 weeks: Bz-423 anti-ssDNA, $738 \pm 120$
$\mathrm{U} / \mathrm{ml}$; control, $809 \pm 175 \mathrm{U} / \mathrm{ml} ; P=0.37$; Bz-423 antidsDNA, $789 \pm 145 \mathrm{U} / \mathrm{ml}$; control, $733 \pm 198 \mathrm{U} / \mathrm{ml}$; $P=0.46 ; \mathrm{Bz}-423 \mathrm{IgG}, 6.7 \pm 0.8 \mathrm{mg} / \mathrm{ml}$; control, $5.5 \pm 1.0$ $\mathrm{mg} / \mathrm{ml} ; P=0.24)$. Similarly, other lymphotoxic drugs can lessen nephritis in NZB/W mice without altering serum anti-DNA levels (26). Since the effects we observe at 3 weeks are small and treatment started after the onset of disease, it is unlikely that they account for the observed disease improvement. Moreover, antiDNA.DNA complexes may not be the principal autoantibody involved in immune complex deposition in $\mathrm{NZB} / \mathrm{W}$ mice (27). Hence, Bz-423 may have a greater effect on the autoantibody subpopulation(s) (and mechanisms) more relevant to disease. Alternatively, functional B cells can mediate disease expression either by serving as antigen-presenting cells or by contributing directly to inflammation (28). If pathogenic NZB/W B cells act in this manner, targeting them could also improve disease without altering anti-DNA titers.

\section{Discussion}

High levels of ROS that accompany the late stages of apoptosis damage macromolecules, whereas physiologic concentrations regulate a range of intracellular signaling pathways (19). Some apoptotic stimuli, e.g., ceramide (29), dexamethasone (30), and TNF- $\alpha$ (31), induce ROS early in their death responses as a means of initiating downstream effector mechanisms such as caspase activation. Similarly, our data demonstrate that the early $\mathrm{O}_{2}{ }^{-}$response induced by interaction of $\mathrm{Bz}-423$ with mitochondria signals an apoptotic program in B lymphocytes. Several B cell-specific signaling pathways respond to ROS and, when so engaged, initiate apoptosis. For example, activation of Bruton's tyrosine kinase by ROS (32) results in phosphorylation of phospholipase $\mathrm{C} \gamma$ (33), which leads to $\mathrm{Ca}^{2+}$-dependent apoptosis. This and related ROS-dependent processes may contribute to the susceptibility of B cells to Bz-423.

Small molecules increase intracellular $\mathrm{O}_{2}{ }^{-}$in a variety of ways, including stimulating release of $\mathrm{O}_{2}{ }^{-}$from oxygenases, inhibiting oxido-reductases, and disrupting MRC activity (34). Inhibitor studies with whole cells pointed to the MRC as the source of $\mathrm{Bz}-423$-induced $\mathrm{O}_{2}^{-}$. Experiments with isolated mitochondria under conditions supporting state 3 respiration demonstrate an $\mathrm{O}_{2}^{-}$response analogous to that in whole cells. Mitochondria did not respond to $\mathrm{Bz}-423$ in state 4 where energy is supplied in the absence of ADP and respiration is minimal. During the transition from state 3 to 4 , the proton-motive force becomes sufficiently high that intermediates competent in one-electron reduction reactions (e.g., ubisemiquinone anion) have extended half-lives, and these conditions favor reduction of $\mathrm{O}_{2}$ to $\mathrm{O}_{2}{ }^{-}$at complex III of the MRC (35). Hence, it is possible that Bz-423 generates $\mathrm{O}_{2}{ }^{-}$by inducing a state-3-to- 4 conversion. Oligomycin, a macrolide natural product 
that binds to the mitochondrial $\mathrm{F}_{0} \mathrm{~F}_{1}$-ATPase (complex $\mathrm{V}$ ), induces a state-3-to-4 transition and generates $\mathrm{O}_{2}^{-}$like $\mathrm{Bz}-423$ (36). Based on these similarities, it is possible that the $\mathrm{F}_{0} \mathrm{~F}_{1}$-ATPase is also the molecular target for $\mathrm{Bz}-423$.

Action of $B z-423$ on autoimmune disease. Traditional chemotherapeutics compromise mitochondrial function indirectly (2). However, several promising investigational agents directly target mitochondria (37). Given this precedent and the relationships between Ramos and GC cell pathology in diseases like lymphoma and SLE, we explored the properties of $\mathrm{Bz}-423$ in vivo. The critical elements of the $\mathrm{Bz}-423$ response in vitro, $\mathrm{O}_{2}^{-}$production and $\mathrm{B}$ cell apoptosis, were observed in $\mathrm{NZB} / \mathrm{W}$ mice after brief dosing. In the $\mathrm{NZB} / \mathrm{W}$ model, pathogenic autoantibodies are the products of activated, class-switched $\mathrm{B}$ cells that emerge from GCs $(9,10,24)$. After 12 weeks, Bz-423 dramatically reduced the number and size of GCs and increased apoptosis in remaining GCs. Treatment did not alter the representation of myeloid or erythroid precursors in bone marrow, decrease peripheral blood counts, or change cytokine production by splenocytes (our unpublished data). Thus, while we cannot exclude other effects, our data are consistent with a model linking Bz-423-mediated disease improvement to apoptosis of GC B cells, an important target for SLE intervention $(10,28)$.

The apoptotic threshold of GC B cells in normal mice is tightly regulated by c-FLIP and signaling through the B cell receptor (BCR), CD40, CD80, and Fas $(38,39)$. Since little is known about how these factors contribute to the survival of autoimmune GC $\mathrm{B}$ cells, it is difficult to deduce why they are susceptible to $\mathrm{Bz}-423$. At present, the most persuasive evidence suggests that the longevity of NZB/W GC B cells is tied to increased expression of costimulatory molecules (38), downregulation of inhibitory receptors (40), and hyporesponsiveness to persistent BCR stimulation (41). With respect to this last point, it is interesting that BCR stimulation also sensitizes B cells to cytotoxic agents (42). Hence, it is possible that receptor ligation contributes to the selectivity of $\mathrm{Bz}-423$. In support of this, we find that anti-IgM sensitized B cells to Bz-423 (our unpublished data). While the molecular basis for BCR sensitization is not fully understood, cross-linking of BCRs can itself elevate intracellular ROS (43). Furthermore, lymphocytes from lupus patients have less reduced glutathione and increased ROS relative to normal cells (44). Thus, the vulnerability of NZB/W GC B cells, in part, may result from an additive effect of $\mathrm{Bz}-423-$ induced $\mathrm{O}_{2}{ }^{-}$and endogenously generated ROS, such that radicals from all sources combine to overwhelm the reducing potential of autoimmune lymphocytes and to trigger apoptosis. However, it is likely that additional factors, like developmental state and local environment, also play a role in modulating cellular susceptibility to Bz-423.
In summary, Bz-423 is a proapoptotic molecule engaging the cell-death machinery in an $\mathrm{O}_{2}{ }^{-}$-dependent manner. Our studies define a novel structure-function relationship for benzodiazepines and point to a new molecular target and pharmacological mechanism that could be of value for the management of SLE. Present studies are directed at identifying this target as a basis for advancing drug-discovery and mechanistic experiments, particularly with respect to selectivity in vivo.

\section{Acknowledgments}

This work was supported by NIH grants AI-47450 and GM-42168 (to G.D. Glick) and GM-50353 (to J.A. Ellman), and by the University of Michigan Multipurpose Arthritis Center.

1. Austin, H.A., and Balow, J.E. 2000. Treatment of lupus nephritis. Semin. Nephrol. 20:265-276.

2. Garrett, M.D., and Workman, P. 1999. Discovering novel chemotherapeutic drugs for the third millennium. Eur. J. Cancer. 35:2010-2030.

3. Druker, B.J., and Lydon, N.B. 2000. Lessons learned from the development of an abl tyrosine kinase inhibitor for chronic myelogenous leukemia. J. Clin. Invest. 105:3-7.

4. Huang, P., and Oliff, A. 2001. Signaling pathways in apoptosis as potential targets for cancer therapy. Trends Cell Biol. 11:343-348.

5. Schreiber, S.L. 2000. Target-oriented and diversity-oriented organic synthesis in drug discovery. Science. 287:1964-1969.

6. Bunin, B.A., Plunkett, M.J., and Ellman, J.A. 1994. The combinatorial synthesis and chemical and biological evaluation of a 1,4-benzodiazepine library. Proc. Natl. Acad. Sci. USA. 91:4708-4712.

7. Beurdeley-Thomas, A., et al. 2000. The peripheral benzodiazepine receptors: a review. J. Neurooncol. 46:45-56.

8. Gregory, C.T., et al. 1987. Identification of a subset of normal B cells with a Burkitt's lymphoma (BL)-like phenotype. J. Immunol. 139:313-318.

9. Portanova, J.P., et al. 1995. An early post-mutational selection event directs expansion of autoreactive B cells in murine lupus. Mol. Immunol. 32:117-135.

10. Shlomchik, M.J., Craft, J.E., and Mamula, M.J. 2001. From T to B and back again: positive feedback in systemic autoimmune disease. Nat. Rev. Immunol. 1:147-153.

11. Kerver, E.D., et al. 1997. In situ detection of spontaneous superoxide anion and singlet oxygen production by mitochondria in rat liver and small intestine. Histochem. J. 29:229-237.

12. Swanson, P.C., and Glick, G.D. 1996. Ligand recognition by anti-DNA autoantibodies. Affinity, specificity and mode of binding. Biochemistry. 35:1624-1633.

13. Belzacq, A.S., et al. 2001. Apoptosis induction by the photosensitizer verteporfin: identification of mitochondrial adenine nucleotide translocator as a critical target. Cancer Res. 61:1260-1264.

14. Doi, T., Motoyama, N., Tokunaga, A., and Watanabe, T. 1999. Death signals from the B cell antigen receptor target mitochondria, activating necrotic and apoptotic death cascades in a murine B cell line, WEHI231. Int. Immunol. 11:933-941.

15. Benov, L., Sztejnberg, L., and Fridovich, I. 1998. Critical evaluation of the use of hydroethidine as a measure of superoxide anion radical. Free Radic. Biol. Med. 25:826-831.

16. Zamzami, N., et al. 1995. Sequential reduction of mitochondrial transmembrane potential and generation of reactive oxygen species in early programmed cell death. J. Exp. Med. 182:367-377.

17. Li, P., et al. 1997. Cytochrome $\mathrm{c}$ and dATP-dependent formation of apaf$1 /$ capase- 9 complex initiates an apoptotic protease cascade. Cell. 91:479-489.

18. Luetjens, C.M., et al. 2000. Delayed mitochondrial dysfunction in excitotoxic neuron death: cytochrome c release and a secondary increase in superoxide production. J. Neurosci. 20:5715-5723.

19. Finkel, T. 1999. Signal transduction by reactive oxygen species in nonphagocytic cells. J. Leukoc. Biol. 65:337-340.

20. Fosslien, E. 2001. Mitochondrial medicine: molecular pathology of defective oxidative phosphorylation. Ann. Clin. Lab. Sci. 31:25-67.

21. Zini, R., Simon, N., Morin, C., Thiault, L., and Tillement, J.P. 1998. Tacrolimus decreases in vitro oxidative phosphorylation of mitochondria from rat forebrain. Life Sci. 63:357-368.

22. Esposti, M.D. 2001. Assessing functional integrity of mitochondria in vitro and in vivo. Methods Cell Biol. 65:75-96.

23. Ohse, T., Nagaoka, S., Arakawa, Y., Kawakami, H., and Nakamura, K. 2001. 
Cell death by reactive oxygen species generated from water-soluble cationic metalloporphyrins as superoxide dismutase mimics. J. Inorg. Biochem. 85:201-208.

24. Munakata, Y., et al. 1998. Somatic mutation in autoantibody-associated $\mathrm{V}_{\mathrm{H}}$ genes of circulating $\operatorname{IgM}^{+} \operatorname{IgD}^{+} \mathrm{B}$ cells. Eur. J. Immunol. 28:1435-1444.

25. Theofilopoulos, A.N., and Dixon, F.J. 1985. Murine models of systemic lupus erythematosus. Adv. Immunol. 37:269-390.

26. Gelfand, M.C., and Steinberg, A.D. 1972. Therapeutic studies in NZB/W mice. Arthritis Rheum. 15:247-255.

27. Lefkowith, J.B., and Gilkeson, G.S. 1996. Nephritogenic autoantibodies in lupus. Current concepts and continuing controversies. Arthritis Rheum. 39:894-903.

28. Owen, T.M.C., Hannum, L.G., Haberman, A.M., Madaio, M.P., and Shlomchik, M.J. 1999. A novel mouse with B cells but lacking serum antibody reveals an antibody-independent role for B cells in murine lupus. J. Exp. Med. 189:1639-1647.

29. Garcia-Ruiz, C., Colell, A., Mari, M., Morales, A., and Fernandez-Checa, J.C. 1997. Direct effect of ceramide on the mitochondrial electron transport chain leads to generation of reactive oxygen species. J. Biol. Chem. 272:11369-11377.

30. Torres-Roca, J.F., et al. 2000. An early oxygen-dependent step is required for dexamethasone-induced apoptosis of immature mouse thymocytes. J. Immunol. 165:4822-4830.

31. Albrecht, H., Tschopp, J., and Jongeneel, C.V. 1994. BCL-2 protects from oxidative damage and apoptotic cell-death without interfering with activation of NFKB by TNF. FEBS Lett. 351:45-48.

32. Qin, S., Stadtman, E.R., and Chock, P.B. 2000. Regulation of oxidative stress-induced calcium release by phosphatidylinositol 3-kinase and Bruton's tyrosine kinase in B cells. Proc. Natl. Acad. Sci. USA. 97:7118-7123.

33. Takata, M., and Kurosaki, T. 1996. A role for Bruton's tyrosine kinase in $\mathrm{B}$ cell antigen receptor-mediated activation of phospholipase C $\gamma 2$.J. Exp. Med. 184:31-40.
34. Siraki, A.G., Pourahmad, J., Chan, T.S., Khan, S., and O’Brien, P.J. 2002 Endogenous and endobiotic induced reactive oxygen species formation by isolated hepatocytes. Free Radic. Biol. Med. 32:2-10.

35. Skulachev, V.P. 1999. Mitochondrial physiology and pathology: concepts of programmed death of organelles, cells and organisms. Mol. Aspects Med. 20:139-184.

36. Korshunov, S.S., Skulachev, V.P., and Starkov, A.A. 1997. High protonic potential actuates a mechanism of production of reactive oxygen species in mitochondria. FEBS Lett. 416:15-18.

37. Costantini, P., Jacotot, E., Decaudin, D., and Kroemer, G. 2000. Mitochondrion as a novel target of anticancer chemotherapy. J. Natl. Cancer Inst. 92:1042-1053.

38. Eijk, M.V., Defrance, T., Hennino, A., and Groot, C.D. 2001. Death-receptor contribution to the germinal center reaction. Trends Immunol. 22:677-682.

39. Tsiagbe, V.K., Inghirami, G., and Thorbecke, G.J. 1996. The physiology of germinal centers. Crit. Rev. Immunol. 16:381-421.

40. Jiang, Y., et al. 1999. Genetically determined aberrant down-regulation of FcyRIBB1 in germinal center B cells associated with hyper-IgG and IgG autoantibodies in murine systemic lupus erythematosus. Int. Immunol. 11:1685-1691.

41. Kozono, Y., Kotzin, B.L., and Holers, V.M. 1996. Resting B cells from New Zealand black mice demonstrate a defect in apoptosis induction following surface IgM ligation. J. Immunol. 156:4498-4503.

42. Lin, C.E., et al. 1998. Apoptosis may be either suppressed or enhanced with strategic combinations of antineoplastic drugs or anti-IgM. Exp. Cell Res. 244:1-13.

43. Fang, W., et al. 1995. Bcl-xL rescues WEHI 231 B lymphocytes from oxidant-mediated death following diverse apoptotic stimuli. J. Immunol. 155:66-75.

44. Gergely, P., Jr., et al. 2002. Mitochondrial hyperpolarization and ATP depletion in patients with systemic lupus erythematosus. Arthritis Rheum. 46:175-190. 